\title{
Health promotion: From malaria control to elimination
}

\author{
M A Groepe, ${ }^{1}$ MSc; J Urbach, ${ }^{2}$ MCom (Economics); H Jooste, ${ }^{3}$ MPA; K W Hlongwana, ${ }^{4}$ MSc; L Baker, ${ }^{5}$ Dip Pharm; E Misiani, ${ }^{6} \mathrm{PhD}$; \\ N T Mayet, ${ }^{7} \mathrm{MB} \mathrm{ChB}, \mathrm{DTM} \& \mathrm{H}$ \\ 'World Health Organization, Pretoria, South Africa \\ ${ }^{2}$ Africa Fighting Malaria, Durban, South Africa \\ ${ }^{3}$ Department of Social Development and Health, Nelspruit, Mpumalanga Province, South Africa \\ ${ }^{4}$ Malaria Research Unit, South African Medical Research Council, Durban, South Africa \\ ${ }^{5}$ Amayeza Information Centre, Johannesburg, South Africa \\ ${ }^{6}$ Malaria Directorate, National Department of Health, Pretoria, South Africa \\ South African Regional Global Disease Detection Centre, National Institute for Communicable Disease, Johannesburg, South Africa
}

Corresponding author: N T Mayet (nataliem@nicd.ac.za)

Here we reflect on the achievement of some of the diverse activities that have brought malaria under control, highlight key challenges and propose specific health promotion interventions required to move South Africa's malaria programme from control to elimination.

S Afr Med J 2013;103(10 Suppl 2):799-800. DOI:10.7196/SAMJ.7444

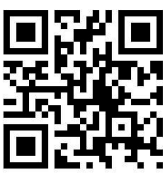

Health promotion, as defined by the Ottawa Charter, is the process of enabling people to increase control over and to improve their health. Three basic strategies for advancing health promotion include; advocacy for health, enabling all people to achieve their full potential and mediating between the different interests in society in the pursuit of health. ${ }^{[1]}$

The Malaria Elimination Communication and Advocacy Strategy 2011 - 2014 of the National Department of Health (NDoH) lists key objectives in achieving malaria elimination: (i) to advocate at political, healthcare worker and community level; (ii) to develop and distribute material on environmental and vector control, chemoprophylaxis, personal protection, signs and symptoms of malaria; and (iii) to ensure patient adherence to treatment and to collaborate with partners. ${ }^{[2]}$ Advocacy, in this instance, refers to the combined individual and social actions designed to gain political commitment, policy support, social acceptance and system support for the programme. ${ }^{[1]}$

\section{Contextual framework}

The earliest reports of health promotion activity in South Africa (SA) dates back to 1904 where Bostock ${ }^{[3]}$ alludes to the use of 'hand-bills dealing with malaria prevention were left at all houses in which cases of malaria had been notified to exist, and in some areas these hand-bills were left at every house. Lectures and magic lantern demonstrations were given by me to societies, institutions, schools; and I feel sure these lectures and demonstrations were a potent agent for good in our campaign'.

Since then there has been considerable and varied activities with respect to advocacy, health promotion and partnership development in the National Malaria Control Programme to date and although there activities are often not documented and subject to the same scientific scrutiny and rigor as clinical trials to demonstrate its net benefit prior to implementation, they form an important pillar of a successful malaria control programme. ${ }^{[4,5]}$

As signatory to the 2000 United Nations (UN) Millennium Development Goals (MDGs) agenda, SA has pledged to work in achieving MDG6 to 'have halted and begun to reverse the incidence of malaria and other major diseases by $2015^{5[6]}$ This commitment set the international political advocacy framework together with a number of other initiatives such as the Roll Back Malaria Partnership (RBM) launched in 1998 by the World
Health Organization (WHO), the UN Children's Fund (UNICEF), the UN Development Programme (UNDP) and the World Bank, in an effort to provide a coordinated global response to the disease.

Regionally, the Lubombo Malaria Protocol of understanding was signed in 1999 between SA, Swaziland and Mozambique and formed the Lubombo Spatial Initiative Development (LSDI). ${ }^{[7]}$ This initiative and the accompanying activities designed to collaborate on key malaria interventions impacted positively on both Swaziland and SA, where their malaria cases decreased by at least $90 \%$. In 2007 , the Africa Union and Southern Africa Development Community (SADC) recommended that SA, among other selected southern African countries, be targeted for malaria elimination.

In 2003, then President Mbeki launched the southern Africa 'Race against Malaria, with the aim of renewing the momentum in southern Africa after decades of low prioritisation. This was the largest malaria awareness intervention ever undertaken by the $\mathrm{NDoH}$ in partnership with SADC countries and key stakeholders of both the private, non-governmental and public sector. ${ }^{[8]}$

The National Malaria Advisory Group, the Medical Research Council and the newly constituted Malaria Elimination Committee play a vital role in providing technical recommendations and advocacy for malaria prevention and control. These policies were translated to relevant operational plans at national, provincial, and district level. They are also included in the integrated development plans of local government particularly in endemic areas. The aim at all levels is to facilitate the planning, implementation, coordination, monitoring and evaluation of health promotion and therefore the role of health promotion differs between the levels. These activities ranged from mass community mobilisation, awareness campaigns, the commemoration of malaria days, and the development of malaria-literate communities and have resulted in SA achieving an indoor residual spraying (IRS) coverage of $>80 \%$ and the completion of spraying before the peak in malaria transmission. The RBM survey conducted in 2005 indicated that SA had by then already more than halved the number of malaria cases. There have been a number of intersectoral collaborations where malaria has been included such as the Integrated Management of Childhood Illness (IMCI) strategy, and the Malaria Collaborative Forum (MCF), a private initiative by a pharmaceutical company that assisted with training and promotional materials. 
There is a paucity of evidence in relation to the impact of the health promotion interventions; however, in a knowledge, attitudes and practices (KAP) study conducted in Bushbuckridge, Mpumalanga Province, in 2008 to determine the key sources of malaria information in relation to the preferred communication channels, it was found that health facilities were the main sources of malaria information where talks and one-onone sessions were conducted. ${ }^{[9]}$ Electronic media, especially the radio, has been very successfully utilised over the years and has contributed to the community's malaria literacy, while printed media is useful as a handout after health education has been conducted, but has been found to have little impact on behaviour change. The study also reflected that posters and pamphlets were the least preferred source of information. ${ }^{[9]}$

\section{Key challenges}

The international, regional and national advocacy at political level and administrative level drove a variety of health promotion activities interventions. However, all of these interventions were run as a vertical programme, in the context of an overburdened healthcare system where tuberculosis and HIV/AIDS interventions had taken the centre stage.

Health promotion, advocacy and partnership interventions are contextualised within the current milieu of: the reduction in malaria literacy in non-endemic areas; the perception of reduced malaria risk among policy makers, healthcare workers and communities; South African cultural diversity and its migrant populations; community perceptions of IRS and of healthcare in general; the vertical nature of the programme; the re-engineered primary healthcare service delivery model $^{[1]}$ and importantly, the limited existing resources required to provide a comprehensive, integrated and standardised advocacy and marketing strategy.

\section{New paradigm}

Advocacy, health promotion, health education, strategic marketing, advertising, and the strengthening of existing partnerships are essential prerequisites in closing the identified gaps in the malaria control programme when moving from control to elimination. ${ }^{[10]}$

To chart the way forward for moving malaria programmes from control to elimination the following recommendations are made:

- Consciously advocate for malaria elimination and the required resourcing to remain on the political agenda and for it to be included on the annual performance plans of national, provincial, district and local government departments.

- Integrate the elimination strategy with the operational plans of other departments e.g. Environmental Affairs, Home Affairs, Tourism, Agriculture, Forestry and Fisheries, Basic Education, Economic Affairs and integrate with primary healthcare within the $\mathrm{NDoH}$.

- Determine the competencies required of staff in endemic and hotspot areas and provide the required training, a malaria tool kit and the necessary infrastructural support.

- Formulate, standardise and simplify core key messaging and ensure that it can be modified and made relevant for specific target audiences.

- Utilise existing community structures and resources e.g. traditional healers, traditional leaders, stockvel groupings as conduits for social dialogue and for dissemination of malaria messages.

- Consider novel approaches of translating the malaria 'elimination message' through industrial theatre or comedy shows for schools, workplaces with the active participation of the audience.

- Core messages are to include the extent of malaria risk, targeted insecticide use; prophylaxis and personal protection during travel to or through a malaria area during transmission season, seeking diagnosis and treatment as soon as malaria signs and symptoms arise can save lives, and adherence to treatment is necessary for self-protection. The community needs to partner inactive surveillance and case investigation.
- Communication activities targeting the traveller, whether recreational or the migrant labourer, will need to be structured and disseminated via border posts, taxi stations, buses and workplaces, using the most appropriate information, education and communication (IEC) materials.

- Further innovative methods of communication such as a smartphone app could be developed and downloaded and used by healthcare professionals and the enquiring traveller. This could incorporate a malaria risk map, pictorial information on the vector, parasite, prophylaxis signs and symptoms, and resources of where further information can be obtained.

- Health advocacy is the responsibility of every single community member and the culture of reporting is to be encouraged either through the formal health structures or via a malaria hotline.

- Existing partnerships are to be strengthened and new partnerships are to be explored with captains of industry, occupational health practitioners, safety officers, the Travel Medicine Society, the South African Medical Association (SAMA), and our neighbours in the region, so that resources can be shared in achieving mutually agreed goals. Formulation of partnerships is a labour-intensive exercise and requires high maintenance, but can be rewarding if there is demonstrated impact of the partnership.

- Gains in the elimination strategy are to be celebrated and communicated to all stakeholders frequently, and the recommendations of all research activity are to be marketed.

- For advocacy or marketing to succeed, it must have an allocated budget and a clearly defined time horizon.

\section{Conclusion}

Effective malaria control is an important precursor to development, with the situation prior to malaria control in SA supporting this view, given the well-documented negative effects of malaria on tourism and development. We need to celebrate our successes.

Health promotion demands a multidisciplinary approach, requiring the combined inputs of social scientists, health workers, communication specialists, policy analysts and others if it is to be successful. Health professionals have a major responsibility to act as advocates for health at all levels of society and partnerships that are voluntary agreements between two or more partners that work cooperatively towards a set of shared outcomes. Evidencebased educational materials should take note of epidemiological, ethnographic, sociological, cultural, historical and political data to be relevant for the setting of implementation. ${ }^{[12]}$

\section{References}

1. World Health Organization. Ottawa Charter for Health Promotion. Geneva: WHO, 1986. http://www. who.int/iris/handle/10665/53166 (accessed 10 August 2013).

who.int/iris/handle/10665/53166 (accessed 10 August 2013).
2. South African NDoH. Malaria Elimination Communication and Advocacy Strategy 2011-2014. 2. South African $\mathrm{NDoH}$.
Pretoria: $\mathrm{NDoH}, 2011$.

Pretoria: NDoH, 2011.
3. Bostock, L. Malaria in South Africa. In: R. Ross, ed. The Prevention of Malaria. Section 55. London: John Murray, 1910:543-548.

Gillon R. Ethics in health promotion and prevention of disease. J Med Ethics 1990;16(4):171-172. [http://dx.doi.org/10.1136/jme.16.4.171]

5. South African NDoH. National Malaria Control Programme Review 2009. Pretoria: NDoH, 2009.

6. South African NDoH. An Overview of South Africa's Progress in the Implementation of the Millennium Development Goals. Governance and Administration Cluster, MDG Presentation, Pretoria: NDoH, 2011. http://www.dpsa.gov.za/docs/presentations/MDG_Presentation_01_Sep_2011.pdf (accessed 12 August 2013).

7. Lubombo Spatial Development Initiative, Malaria Control Programme. http://www.malaria.org.za/ 1sdi/Background/background.html (accessed 10 August 2013).

8. Mbeki T. The Race Against Malaria. 3 April 2003. http://www.thepresidency.gov.za/pebble.asp?relid=3010 Mbeki T. The Race Against
(accessed 10 August 2013)

9. Hlongwana KW, Zitha MA, Mabuza AM, Maharaj R. Knowledge and practices towards malaria 9. Hlongwana KW, Zitha MA, Mabuza AM, Maharaj R. Knowledge and practices towards malaria
amongst residents of Bushbuckridge, Mpumalanga, South Africa. Afr J Prim Health Care Fam Med 2011;3(1). [http://dx.doi.org/10.4102/phcfm.v3i1.257]

10. World Health Organization. Malaria Programme Reviews: A Manual for Reviewing the Performance of Malaria Control and Elimination Programmes. Geneva: WHO, 2010. http://www.who.int/malaria/ publications/atoz/whomprmalariaprogramperformancemanual/en/index.html (accessed 10 August 2013). 1. South African NDoH. Provincial Guidelines for the Implementation of the Three Streams of Primary Health Care Re-engineering. Pretoria: $\mathrm{NDoH}, 2011$.

2. Smith, BJ, Tang KC, Nutbeam, D. WHO Health Promotion Glossary: New terms. Health Promot Int 2006;21:340-345. [http://dx.doi.org/10.1093/heapro/dal033] 